\title{
Phase separation driven by interchangeable properties in the intrinsically disordered regions of protein paralogs
}

Shih-Hui Chiu ${ }^{1}$, Wen-Lin Ho ${ }^{1}$, Yung-Chen Sun ${ }^{1}$, Jean-Cheng Kuo ${ }^{1}$, and Jie-rong Huang $1,2,3, *$

${ }^{1}$ Institute of Biochemistry and Molecular Biology, National Yang Ming Chiao Tung University, No. 155 Section 2, Li-nong Street, Taipei, Taiwan

${ }^{2}$ Institute of Biomedical Informatics, National Yang Ming Chiao Tung University, No. 155 Section 2, Li-nong Street, Taipei, Taiwan

${ }^{3}$ Department of Life Sciences and Institute of Genome Sciences, National Yang Ming Chiao Tung University, No. 155 Section 2, Li-nong Street, Taipei, Taiwan

To whom correspondence should be addressed: jierongh@nycu.edu.tw 


\begin{abstract}
Paralogs, arising from gene duplications, increase the functional diversity of proteins. Protein functions in paralog families have been extensively studied, but little is known about the roles of intrinsically disordered regions (IDRs), even though more than half of eukaryotic proteins have them. Using the RNA-binding protein Musashi family as an example, we applied multiple structural techniques and phylogenetic analysis to show how members in a paralog family have evolved their IDRs to different physicochemical properties but converge to the same function. In this example, the lower prion-like tendency of Musashi-1's IDRs, rather than Musashi-2s', is compensated by its higher $\alpha$-helical propensity to assist their assembly. Without a folded structure to restraint, IDRs' sequences mutate faster along with evolution. No matter how fast they change, our work suggests that IDRs evolve different traits to a converged function, such as liquid-liquid phase separation.
\end{abstract}




\section{Introduction}

The myriad functions of proteins have arisen from recurrent gene duplications, which create paralogs in a genome (Ohno 1970). The redundant copy, if not silenced as a pseudogene, enhances survival, and many duplicated genes mutate toward new functions (Wilson 1899). The paralog $\alpha$ - and $\beta$-globin chains that form the haemoglobin tetramer are a textbook example (Lodish, et al. 2016): The existence of two copies of the $\alpha$-globin gene (HBA1 and $H B A 2)$ reduces the effect of $\alpha$-thalassemia, but not the $\beta$-thalassemia, which results from the loss of $\beta$-globin encoding in only $H B B$; These paralogs, which have evolved from an ancestral oxygen-binding globin, work cooperatively in their new role of oxygen transport (Lodish, et al. 2016). Although protein paralogs have been studied for decades, the paralogs of intrinsically disordered proteins (IDPs) or proteins with intrinsically disordered regions (IDRs), accounting for more than half of the eukaryotic proteome (Uversky and Dunker 2010), remain largely unexplored.

Our particular interest is in RNA-binding proteins (RBPs), which are at the core of gene regulation and RNA metabolism and whose dysfunction is implicated in many diseases (Gebauer, et al. 2020). In addition to the common feature of having RNA interacting motifs (Dominguez, et al. 2018), RBPs often contains IDRs (Varadi, et al. 2015; Zagrovic, et al. 2018). The role of these IDRs had been unclear until it was recently shown that they can organize cellular structures without a lipid membrane (Kwon, et al. 2013). These membraneless organelles, which assemble via liquid-liquid phase separation (LLPS), have since been extensively studied and have become a working model of spatiotemporal control for many cellular functions (Alberti and Hyman 2021). Although some RBPs' IDRs have been reported involved in forming membraneless organelles (such as RNA or stress granules), a large proportion of them are still of unknown function. The RBPs themselves are often paralogs, many of which share highly conserved RNA binding domains but with different IDRs. In the manually curated list of RBPs (1,542 RBPs in total) (Gerstberger, et al. 2014), more than $22 \%$ $(341 / 1542)$ are annotated in one of the paralog families based on the OrthoMCL database (Li, et al. 2003), and more than $48 \%$ are paralogs (749/1542) as defined in the original census study (Gerstberger, et al. 2014) (see supplementary dataheets S1 and S2 for the lists, Supplementary Material online). These paralogs have similar folded domains, hinting at similar RNA recognition mechanisms, whereas many disordered regions have greater sequence diversity.

To understand the different properties of IDRs in paralogs, we focus on those twomember families and found that the Musashi protein family is a suitable example for this purpose. The Musashi gene was originally identified in the fruit fly (Drosophila 
melanogaster), responsible for sensory organ development (Nakamura, et al. 1994) and is highly conserved in animals (Sakakibara, et al. 1996; Hirota, et al. 1999; Kawashima, et al. 2000; Yoda, et al. 2000; Shibata, et al. 2012). In mammals, the two Musashi paralogs are translational regulators of cell fate and are involved in maintaining the stem-cell state, differentiation, and tumorigenesis (Okano, et al. 2002). In addition to their role in neural stem cell development (Sakakibara, et al. 1996; Sakakibara, et al. 2002; Shibata, et al. 2012), they also regulate several types of cancer (Lagadec, et al. 2014; Fox, et al. 2015). The C-terminal domain (an IDR) is critical for forming chemoresistant stress granules in glioblastoma (Chiou, et al. 2017) and colorectal cancer cells (Chen, et al. 2018). Musashi proteins cannot join stress granules without it (Chiou, et al. 2017; Chen, et al. 2018). Furthermore, the toxic oligomers formed by Musashi proteins have been implicated in Alzheimer's disease (Sengupta, et al. 2018; Montalbano, et al. 2020). We noticed through sequence analysis that the IDRs of Musashi-1 and -2 have different physical properties, which are conserved among vertebrate orthologs. Here, our data suggest that the decreased prion-likeness of one paralog is compensated in assembly formation by an increase in $\alpha$-helical propensity. These results show how different properties may have evolved in IDR paralogs for the same biophysical mechanism.

\section{Results}

The IDRs of the Musashi family have different prion propensities

We used the primary sequence of the folded domain of fruit fly Musashi (residues 29-195, a predicted RNA recognition motif(RRM)) to identify orthologs in the UniProt database (i.e. ignoring its IDR). We selected the model organisms (Hedges 2002) (listed in supplementary table S1, Supplementary Material online) with similar RRM sequences and constructed a phylogenetic tree using their full-length sequences (fig. $1 A)$. The nematode and fruit fly have only one Musashi gene, but vertebrates have two paralogs (fig. 1A). The C-terminal half of all these proteins are intrinsically disordered (purple bars in fig. $1 A$; supplementary fig. S1, Supplementary Material online). Note that the IDR was not used to identify orthologs but is a common feature of all Musashi proteins. Results suggest the IDR is involved in Musashi-1 joining stress granules (Chiou, et al. 2017; Chen, et al. 2018), and many other stress-granule-related proteins have a prion-like domain. We therefore used the PLAAC algorithm (Lancaster, et al. 2014) to predict prion-likeness. Although all the IDRs of Musashi-2 in the vertebrates and lower animals are prion-like, the IDRs of Musashi-1 orthologs are less so (fig. 1A). Fig. $1 B$ compares the human Musashi paralogs, where the IDRs cover residues 237362 (Msi-1C) and 235-328 (Msi-2C) (red box). We separated all Musashi protein 
sequences into IDRs and RRMs based on their alignment to the corresponding human ortholog (fig. $1 B$ and supplementary fig. S2, Supplementary Material online). The RRMs in Musashi-1 and Musashi-2 have very similar amino-acid compositions (fig. $1 C, D$; supplementary table S2, Supplementary Material online). On the contrary, although the IDRs of the two paralogs have many glycines, prolines, and alanines (fig. $1 C$, supplementary table S2, Supplementary Material online), their amino-acid sequences differ substantially (fig. $1 D$, lower panel). Sorting the amino acids in terms of prion-forming propensity (Lancaster, et al. 2014) reveals that the Musashi-1 orthologs have fewer prion-promoting amino acids, such as glutamine and asparagine, than Musashi-2 orthologs do. This amino acid composition analysis reinforces the suggestion that Musashi-2 is more prion-like (fig. 1D).

The disordered regions of Musashi proteins have a polyalanine region that forms an $\alpha$ helix.

We purified the human Musashi proteins' IDRs to investigate their differences experimentally (supplementary fig. S3, Supplementary Material online). Their circular dichroism (CD) spectra show that they are mostly unstructured (fig. $2 A$ ). The CD patterns of Msi-1C and Msi-2C at pH 5.5, 283K (the conditions at which the proteins were most stable and soluble, see below), are similar overall but differ slightly around $220 \mathrm{~nm}$ (indicated by an arrow in fig. $2 A$ ), hinting at a potential difference in $\alpha$-helicity (Uversky 2002; Greenland, et al. 2018). We assigned the NMR chemical shifts of the two IDRs to obtain residue-specific structural propensities (BMRB accesses: 51207 and 51208 ). In the ${ }^{15} \mathrm{~N}$-edited heteronuclear single-quantum coherence (HSQC) spectra (fig. $2 B$ ), most of the amide proton signals are within $1 \mathrm{ppm}$, confirming the disordered nature of these domains (Prestel, et al. 2018). Nevertheless, both regions contain a stretch of residues in which the secondary chemical shifts (the differences between the measured chemical shifts and random-coil values) are positive for $\mathrm{C} \alpha$ and $\mathrm{C}$ ' atoms and negative for $\mathrm{C} \beta$ atoms, indicating a propensity to form an $\alpha$-helix (fig. $2 C$ ). The deviations from random-coil values are smaller for Msi-2C than for Msi-1C (fig 2C). $\delta 2 \mathrm{D}$ predictions (Camilloni, et al. 2012) based only on chemical shifts (with no missing assignments in the $\alpha$-helical region) indicate that the $\alpha$-helical propensity of Msi-1C is higher than Msi-2C's (residue-specific values up to $\sim 50 \%$ vs up to $\sim 20 \%$; red bars in fig. 2D). These $\alpha$-helical forming regions correspond to the polyalanine stretches of the two proteins (fig. $2 D, E$ ). Msi-1C contains an eight-alanine-repeat whereas Msi-2C's polyalanine stretch is interrupted (and its $\alpha$-helical propensity reduced) by a $\beta$-sheetpromoting valine (Levitt 1978; Pace and Scholtz 1998). Importantly, the respectively continuous and disrupted polyalanine tracts are conserved in Musashi-1 and Musashi2 orthologs (fig. $2 E$ and supplementary fig. S2, Supplementary Material online). 
The non-conserved regions flanking the polyalanine tracts tune their $\alpha$-helical propensity

Musashi-1 has two residue stretches not found in Musashi-2 around the polyalanine tract (denoted Seq1 and Seq2; fig. $3 A$ ). We created three constructs without Seq1, Seq2, or both, to investigate their effect (fig. $3 B ; \Delta$ Seq1, $\Delta$ Seq2, and $\Delta \mathrm{Seq} 1 \Delta \mathrm{Seq} 2)$. The CD patterns of these variants are similar to those of Msi-1C (fig. $3 C$ ) and in the ${ }^{15} \mathrm{~N}-{ }^{1} \mathrm{H}$ HSQC spectra, most peaks overlap with those of Msi-1C. However, there are pronounced changes between Seq1 and Seq2 (fig. 3D). The adjacency of these regions to the mutation sites is not the only cause because residues further away from the polyalanine region show smaller chemical shift perturbations than those within the polyalanine region (as highlighted in fig. $3 D$ as an example and quantified in fig. $3 E$ ). These changes show systematic patterns with downfield chemical shifts in the proton and nitrogen dimensions for $\Delta$ Seq 1 and the opposite trend for $\Delta$ Seq 2 (fig. $3 D, E$ ), indicating a shifting equilibrium between different conformations in the fast exchange regime. To confirm this, we assigned the ${ }^{13} \mathrm{C}$ chemical shifts of the three variants (BMRB accesses: 51204, 51205, and 51206; supplementary fig. S4A, Supplementary Material online), which are mostly similar, except those close to the polyalanine region (supplementary fig. $4 B$, Supplementary Material online). We calculated the secondary chemical shift difference of $C \alpha$ and $C \beta$ atoms $(\Delta \delta C \alpha-\Delta \delta C \beta$; which minimizes the error from chemical shift referencing) and compared these values in the variants to those in the wild type $(\Delta(\Delta \delta \mathrm{C} \alpha-\Delta \delta \mathrm{C} \beta)$; fig. $3 F)$. Since larger $(\Delta \delta \mathrm{C} \alpha-$ $\Delta \delta \mathrm{C} \beta$ ) values indicate a stronger $\alpha$-helical tendency, these results indicate that the wildtype's $\alpha$-helical propensity is lower than $\Delta$ Seq1's but higher than $\Delta$ Seq2's. The estimated $\alpha$-helical propensities (using the $\delta 2 \mathrm{D}$ program) are up to $6 \%$ higher than in the wild type for $\Delta$ Seq1 but up to $5 \%$ lower than in the wild type for $\Delta$ Seq2 (fig. $3 G$ ). These results are in keeping with the indistinguishable CD data because, relative to the entire length of the sequences, the difference in $\alpha$-helical propensity is negligible $(<10 \%$ difference over just $10 \%$ of the sequence). The $\Delta$ Seq $1 \Delta$ Seq 2 variant has a similar predicted secondary structural population as the wild type (fig. $3 G$ ). These results indicate that $\alpha$-helical propensity around the polyalanine stretch is altered by these non-conserved variations in primary sequence with, in order of $\alpha$-helical propensity, $\Delta$ Seq $1>$ wild-type $\approx \Delta$ Seq $1 \Delta$ Seq $2>\Delta$ Seq 2 . Notably, there are no species in which Seq1 or Seq2 are missing alone, hinting that this $\alpha$-helical propensity may have been fine-tuned.

\section{The polyalanine region promotes Musashi-1 assembly}

Since Msi-1C and Msi-2C differ in $\alpha$-helical propensity and the regions in which they differ most change this propensity, we investigated the effects of removing the polyalanine stretch between Seq1 and Seq2. The CD curve of this variant, denoted 
$\triangle$ SeqA, differs from that of Msi-1C around $220 \mathrm{~nm}$ (fig. $4 A$ ), in a similar manner as Msi-2C's does (fig. 2A), indicating a loss of $\alpha$-helicity. Except for the truncation sites, the HSQC spectrum overlaps well with Msi-1C's, indicating no further structural change (fig. $4 B, C$ ). Because NMR and CD signal intensities did not correlate with protein concentrations and Musashi proteins have been reported to form stress granules (Chiou, et al. 2017; Chen, et al. 2018), we used optical microscopy and indeed observed condensates in each case (fig. $4 D$ and supplementary fig. S5, Supplementary Material online). At pH 5.5, the condensates were all spherical, and fluorescence recovery after photobleaching (FRAP) measurements on proteins labelled with a fluorescent probe (Cy3-NHS) showed that the condensates are dynamic (fig. 4E). At $\mathrm{pH} 6.5$, on the contrary, the condensates observed for Msi-1C were irregular and showed no fluorescence recovery (fig. $4 D, E$ and supplementary fig. S6, Supplementary Material online), whereas, for Msi-2C and $\Delta \mathrm{Seq}$, the condensates remained spherical and dynamic (fig. 4D, supplementary fig. S6, Supplementary Material online). We also noticed that the level of fluorescence recovery varied between samples (grey lines in fig. $4 E$ ), indicating that the condensates undergo very rapid sol-gel transitions (fig. $4 E$, supplementary fig. S6, Supplementary Material online), especially for Msi-1C at $\mathrm{pH}$ 5.5 , (i.e., quantifying the level of recovery would not be informative). Rapid gelation has also been reported for other proteins (Molliex, et al. 2015; Patel, et al. 2015; Hardenberg, et al. 2020). Therefore, to compare the aggregation tendency, we prepared $20 \mu \mathrm{M}$ samples at $\mathrm{pH} 5.5$ and 6.5 and incubated them at room temperature for different periods. We then centrifuged the samples (at $\sim 12,000 \times \mathrm{g}$, to remove large aggregates but leave small condensates in the supernatant, as confirmed by microscopy) and measured the concentration of the soluble fraction. At $\mathrm{pH} 5.5$, the proteins remained mostly soluble for all incubation times (fig. $4 F$, as determined by the absorbance at 280 nm; confirmed by SDS-PAGE gel, supplementary fig. S6, Supplementary Material online). At pH 6.5, nearly all Msi-1C molecules aggregated within $1 \mathrm{~h}$ of incubation but Msi-2C, and to a lesser extent, the $\Delta$ SeqA construct remained partially soluble for longer (at least $24 \mathrm{~h}$; fig. $4 F$ ). These results suggest that the polyalanine region in Msi$1 \mathrm{C}$ promotes aggregation. Fig. $4 G$ shows an energy landscape representation of the metastable nature of LLPS in these proteins (Hardenberg, et al. 2020). Their three main states are the monomeric form, the LLPS state (dynamic), and the aggregate form. At $\mathrm{pH} 5.5$, the energy barrier between LLPS and aggregation is high, leaving Msi-1C trapped in the dynamic condensate. At $\mathrm{pH} 6.5$, however, the energy barrier is lower, and Msi-1C aggregates quickly. However, at the same $\mathrm{pH}, \Delta$ SeqA remains in the dynamic soluble state for longer, indicating that removing the $\alpha$-helical region restores the energy barrier between the LLPS and aggregate states. These results suggest that although Msi-1C is less prion-like, its stronger $\alpha$-helical propensity promotes assembly, 
regardless of the "price paid" in terms of aggregation (Patel, et al. 2015; Itakura, et al. 2018).

\section{Discussion}

Various properties contribute to functional assembly in IDRs

Without the constraints of a fixed shape, the IDRs in a paralog family can evolve more freely than structured regions, either gaining new functions or compensating for lost ones. The results obtained here for the Musashi protein family suggest IDRs may have evolved different means of functional assembly. Musashi-1 is found in stress granules, but its IDR has fewer prion-promoting amino acids than many others with this property (e.g., FUS, TDP-43, hnRNPA1). However, our results suggest that the stronger $\alpha$-helical propensity of Musashi-1's polyalanine stretch may assist its assembly, as polyalanine tracts are known to contribute to protein self-assembly (Polling, et al. 2015). On the other hand, Musashi-2's IDR is sufficiently prion-like for assembly despite a lower $\alpha$-helical propensity (fig. 4).

Our results also explain a number of biological observations. Musashi proteins are overexpressed for cell renewal and stemness maintenance (Fox, et al. 2015). Although certain cell types express one or other Musashi paralogs (e.g., Musashi-2 in hematopoietic stem cells (Kharas, et al. 2010)), they are functionally redundant when they appear together. For example, Musashi-2 compensates for the proliferation of neural progenitor cells in Musashi-1 double-knockout mice (Sakakibara, et al. 2002); both Musashi proteins promote colorectal cancer cell growth through the same signaling pathway ( $\mathrm{Li}$, et al. 2015); complete loss of visual function in photoreceptor cells is only observed in Musashi-1,2 double knockout mice (Sundar, et al. 2020). The IDRs' different physical properties compensate for the loss of the other, but these differences (e.g., $\alpha$-helix dominant vs more prion-like) may nevertheless lead to specific interaction mechanisms, for instance, tau protein only interacts with Musashi1 for transportation into nuclei, whereas tau's interaction with Musashi-1 or -2 leads to different pathological stages in tauopathies (Montalbano, et al. 2020).

\section{$\alpha$-helicity is commonly involved in promoting self-assembly}

Polyalanine sequences may be a common evolved trait through which RBPs assemble or join membraneless organelles. For example, a sufficiently long polyalanine tract in its IDR enhances the subnuclear targeting properties of RBM4 (RNA binding motif 4) (Chang, et al. 2014). Indeed, with glutamine and asparagine, alanine is one of the most frequently repeated amino acids in proteins, and polyalanine stretches promote self-assembly (Polling, et al. 2015). Several other amino acids are also helix-promoting, 
such as methionine, leucine, and glutamine (Pace and Scholtz 1998). In the IDR of the extensively studied TDP-43, for example, the "AMMAAAQAALQ" amino acid motif has a strong tendency to form an $\alpha$-helix, promoting condensation (Conicella, et al. 2016; Li, Chen, et al. 2018; Li, Chiang, et al. 2018) regardless of its short polyalanine tract. We rapidly searched for this trait in the proteome using a simple scoring function based on amino acid $\alpha$-helical propensity values derived from our experimental observations and consensus studies (fig. 5A) (Levitt 1978; Pace and Scholtz 1998). Using these tentative scores and aggregating for repeats (examples shown in fig. $5 B$ ), we calculated the portion of all IDRs in the human proteome and in RBPs/mRBPs in which the aggregated $\alpha$-helix score reaches $1.5,2.0$ or 2.5 (fig $5 C$ ). Our analysis shows that helix-promoting stretches are more common in RBPs, especially mRBPs, than in the general human proteome (fig. 5C). The difference is significantly higher than the deviation of 100 random selections of 1542 or 689 proteins from the human proteome (the sample sizes considered for the RBPs and mRBPs, respectively). We attribute the results to that RBPs often join biomolecular condensates for their functions (Wiedner and Giudice 2021).

\section{When did Musashi IDRs diverge?}

The IDRs of Musashi proteins may have respectively gained $\alpha$-helicity or become less prion-like. Which came first? The answer is perhaps hidden in the primary sequence. Using the RRM from D. melanogaster Musashi once again, we searched for orthologs in the phylum Chordata, a higher taxonomic rank than the subphylum of vertebrates in which the Musashi paralogs arise (fig. 1A). Amphioxus (the lancelet), a model organism for primitive chordates, has a Musashi homolog which does not have a long polyalanine tract but is prion-like, similar to that of the nematode and fruit fly (fig. $6 A$ and $B$; supplementary fig. S7, Supplementary Material online). This suggests that the Musashi paralogs may have arisen after the appearance of chordates. In ghost sharks indeed, primitive vertebrates with the two Musashi paralogs, Musashi-1 has a polyalanine tract that is not prion-like (fig. $6 C$; supplementary fig. S7, Supplementary Material online), whereas interestingly, Musashi-2 has a polyalanine tract and is prionlike. Collectively, these results suggest that the polyalanine stretch may have appeared in the paralogs alongside primitive prion propensity before Musashi-l lost its prionpromoting amino acids, whereas a disruption in the $\alpha$-helical region reduced Musashi2's tendency to self-assemble.

\section{"Distant relatives" in the Musashi family}

We searched for sequences similar to Musashi-1's in the human proteome. Other than Musashi-2 (71.3\% identity), the top hits are DAZ-associated protein 1 (DAZAP1, 42.6\%), several heterogeneous nuclear ribonucleoproteins (hnRNP A0, A1, A2...; 
39.0 42.5\%) and TDP-43 (34.9\%) (fig. 6D). This is in agreement with the RBP census study (Gerstberger, et al. 2014), in which the two Musashi proteins, TDP-43, and DAZAP1 were grouped in a paralog family based on sequence similarity. Studies of the low complexity IDRs of hnRNP A1 and A2 are pioneering examples in the emerging field of protein LLPS (Kato, et al. 2012; Molliex, et al. 2015; Xiang, et al. 2015). This low complexity region is also a good model for studying LLPS theory: for example, the number and distribution pattern of aromatic residues in this domain determine its phase separation behavior (Martin, et al. 2020). TDP-43's IDR is another extensively studied example. TDP-43's IDR differs from hnRNP A1 or A2's in that as well as being prionlike, it encompasses a short $\alpha$-helical region that contributes to self-association and LLPS (Conicella, et al. 2016; Li, Chen, et al. 2018). Furthermore, TDP-43's LLPS is mediated by just a few aromatic residues (Li, Chiang, et al. 2018), a feature we have attributed to the presence of the $\alpha$-helix, which promotes intermolecular contacts $(\mathrm{Li}$, Chiang, et al. 2018) (fig. 6D). The present study adds the biophysical properties of the two Musashi proteins to existing information on this distantly related protein family. Although DAZPA1 has been far less studied than other members, its C-terminal domain is critical to its function in interacting with eIF4G (Smith, et al. 2011) and potentially interacts with many RBPs, including hnRNP A1(Yang, et al. 2009). Moreover, bioinformatic analysis shows that this protein's C-terminal domain is disordered and prion-like, with many aromatic residues but no predicted $\alpha$-helix, similar to that of hnRNP A1 (fig. 6E, $F$ and supplementary fig. S8, Supplementary Material online).

In summary, we suggest that IDRs function through a variety of different traits, in keeping with François Jacob's view that "evolution does not produce novelties from scratch" (Jacob 1977). He was referring to the diversity of all lifeforms but his words also apply to IDRs, which acquire new functions through evolutionary "tinkering" (Jacob 1977) between prion-likeness, $\alpha$-helicity, aromatic residues, etc. Our work could be used as a template to investigate IDRs in other paralogs and how their functions have diversified or been preserved during evolution.

\section{Materials and methods}

\section{Sequence analysis}

The primary sequences were obtained from UniProt with the associated entries listed in Supplementary Table 1. Levels of structural disorder and prion-likeness were respectively analyzed with the PONDR (Obradovic, et al. 2005) and PLAAC (Lancaster, et al. 2014) webservers. The phylogenic trees were construed using the neighborjoining method in MEGA X (Kumar, et al. 2018). $\alpha$-helical propensities were predicted 
using RaptorX (Yang, et al. 2018). For the rapid search for helix-forming sequences, all human protein sequences were retrieved from UniProt (UniProtKB_2021_01, download date: 2021/02/06, 20396 sequences in total) and separated into disordered and ordered regions based on PONDR predictions. The $\alpha$-helical scoring of the sequences was done using in-house scripts.

\section{DNA constructs}

The Msi-1C construct in this study (residues number 237 to 362) was cloned from a previous longer construct (194-362)(Chen and Huang 2020). We removed a strongly hydrophobic region reported to contain binding sites for PABP and GLD2 (Fox, et al. 2015), which is irrelevant to the present study and whose removal improves the protein's solubility. The $\Delta$ Seq1, $\Delta$ Seq2, $\Delta$ Seq1 $\Delta$ Seq2, and $\Delta$ SeqA constructs were prepared with designed primers (supplementary table S3, Supplementary Material online). Msi-2 cDNA (residues 234-328, according to the alignment with Msi-1C) was purchased from OriGene. All these variants were constructed in a pET21 vector backbone with a hexa-histidine tag on the C-terminus of the expressed protein. All constructs were fully sequenced.

\section{Protein expression and purification}

All constructs were purified using the same protocol, which has been described in detail elsewhere (Chen and Huang 2020). In short, transformed E. coli BL21(DE3) cells were grown at $37{ }^{\circ} \mathrm{C}$ until the O.D. reached 0.6 and were induced with a final concentration of $1 \mathrm{mM}$ isopropyl- $\beta$-D-1-thiogalactopyranoside at $25{ }^{\circ} \mathrm{C}$ overnight. The cells were harvested and lysed by sonication. After centrifugation, the inclusion bodies were dissolved using $20 \mathrm{mM}$ Tris buffer at $\mathrm{pH} 8$ with $8 \mathrm{M}$ urea (buffer A). After a second centrifugation, the supernatant was filtered with a $0.45 \mu \mathrm{m}$ filter and loaded onto a nickel-charged immobilized metal affinity chromatography column (Qiagen). After washing with ten column volumes of buffer A, the samples were eluted with 5 column volumes of buffer B (buffer A with $500 \mathrm{mM}$ imidazole). The eluted samples were acidified with trifluoroacetic acid (down to $\mathrm{pH} \sim 3$ ), loaded onto a $\mathrm{C} 4$ reversephase column (Thermo Scientific Inc.), and eluted with a gradient of acetonitrile (from 0 to $100 \%$, mixed with triple-distilled water) by HPLC and then lyophilized. The lyophilized samples were stored in a dry cabinet until use. For all experiments, powder samples were dissolved in $20 \mathrm{mM}$ MES-NaOH at $\mathrm{pH} 5.5$ or 6.5. The protein concentration was determined from the absorbance at $280 \mathrm{~nm}$ measured with a Nanodrop spectrometer (Thermo Scientific Inc.).

\section{Circular dichroism spectroscopy}

Circular dichroism spectra were recorded using an AVIV model 410 spectropolarimeter with a $0.1 \mathrm{~mm}$ cuvette. Data were collected between $190 \mathrm{~nm}$ and 
$260 \mathrm{~nm}$ with an interval of $1 \mathrm{~nm}$. Ten measurements were co-added for each data point. All spectra were recorded at $283 \mathrm{~K}$ and the samples were kept in a water bath at $283 \mathrm{~K}$ between measurements. All experiments were performed in triplicate.

NMR spectroscopy, chemical shift assignment, and data analysis

${ }^{15} \mathrm{~N}$-edited HSQC spectra were recorded using the standard pulse sequence with WATERGATE solvent suppression (Bodenhausen and Ruben 1980; Piotto, et al. 1992). Chemical shifts were assigned using standard HNCA, $\mathrm{HN}(\mathrm{CO}) \mathrm{CA}$, HNCO, $\mathrm{HN}(\mathrm{CA}) \mathrm{CO}, \mathrm{CBCA}(\mathrm{CO}) \mathrm{NH}$, and $\mathrm{HNCACB}$ experiments acquired with non-uniform sampling (25\%) (Hyberts, et al. 2009; Hyberts, et al. 2012). All data were recorded using a Bruker AVIII $600 \mathrm{MHz}$ spectrometer with a cryogenic probe.

The data were processed using NMRPipe (Delaglio, et al. 1995). Chemical shifts were assigned using the automated assignment scheme (Lee, et al. 2009) implemented in NMRFAM-Sparky (Lee, et al. 2015), and then confirmed manually. Secondary chemical shift analysis was performed using Kjaergaard et al.'s database of randomcoil shifts (Kjaergaard, et al. 2011). Secondary structure populations were estimated using $\delta 2 \mathrm{D}$ (Camilloni, et al. 2012). No chemical shifts were missing around the critical $\alpha$-helical region, such that the secondary structure estimates for the constructs were made using the same number of chemical shifts.

\section{Microscopy}

Protein samples were loaded onto mPEG-passivated slides (Alberti, et al. 2018). Micrographs were collected using an Olympus BX51 microscope with a 40× longworking-distance objective lens.

\section{Microscopy and fluorescence recovery after photobleaching (FRAP) experiments}

For the fluorescent dye labeling, $2.5 \mathrm{mg}$ lyophilized protein was dissolved in 0.1 $\mathrm{M}$ sodium phosphate with $6 \mathrm{M}$ guanidine hydrochloride at $\mathrm{pH} 8.3$ and mixed with $15.6 \mathrm{mM}$ ( $1 \mathrm{mg}$ in total) of Cy3-NHS (Lumiprobe) overnight at room temperature. The excess Cy3-NHS was removed and the buffer was exchanged with a $20 \mathrm{mM}$ MES buffer at pH 5.5 using a PD-10 column (GE Healthcare). The typical labeling efficiency, determined from the ratio of extinction coefficients measured for the protein and the fluorescence dye (Cy3: $150,000 \mathrm{M}^{-1} \mathrm{~cm}^{-1}$ at $\left.550 \mathrm{~nm}\right)$ was $\sim 20 \%$. The Cy3-labeled sample was aliquoted, flash-frozen, and stored at $-80^{\circ} \mathrm{C}$ before usage.

The lyophilized samples were dissolved in $20 \mathrm{mM}$ MES buffer at $\mathrm{pH} 5.5 \mathrm{or} \mathrm{pH}$ 6.5 and mixed with a Cy3-labeled sample with a final concentration of $20 \mu \mathrm{M}$ and $\sim 1$ $\%$ Cy3-labeled protein. The samples were then loaded onto ultraclean coverslips and observed with an iLas multi-modal of total internal reflection fluorescence (Roper Scientific, Inc.)/spinning disk (CSUX1, Yokogawa) confocal microscope (Ti-E, Nikon) 
equipped with 100X1.49NA plan objective lens (Nikon). The condensates were bleached with a $561 \mathrm{~nm}$ laser. Images were acquired at one-second intervals using an Evolve EMCCD camera (Photometrics) and were analyzed using the Metamorph software (Molecular Devices, LLC).

Aggregation assays

Lyophilized samples were dissolved in $20 \mathrm{mM}$ MES buffer at $\mathrm{pH} 5.5$ or $\mathrm{pH} 6.5$ and stored at room temperature for different times. The samples were then centrifuged at $12000 \times \mathrm{g}$ for $5 \mathrm{~min}$. Supernatant concentrations were determined using a NanoDrop spectrometer. Supernatant samples were also analyzed using SDS-PAGE gels to confirm the amount of protein present. All experiments were performed in triplicate.

\section{Acknowledgements}

The authors thank Prof. Won-Jing Wang (NYCU) for access to the microscope, Academia Sinica High-Field NMR Center for technical support (HFNMRC is funded by Academia Sinica Core Facility and Innovative Instrument Project (AS-CFII-108112)), Chen-Yu Hung for microscope setting, and Dr. Tsai-Chen Chen for initial work on this project. This work was supported by the Ministry of Science and Technology of Taiwan (109-2113-M-010-003 and 110-2113-M-A49A-504-MY3 to J.R.H. and 1092326-B-010-002 -MY3 to J.C.K.).

\section{Data Availability}

All data generated or analyzed in this study are in the Supplementary Material online. 

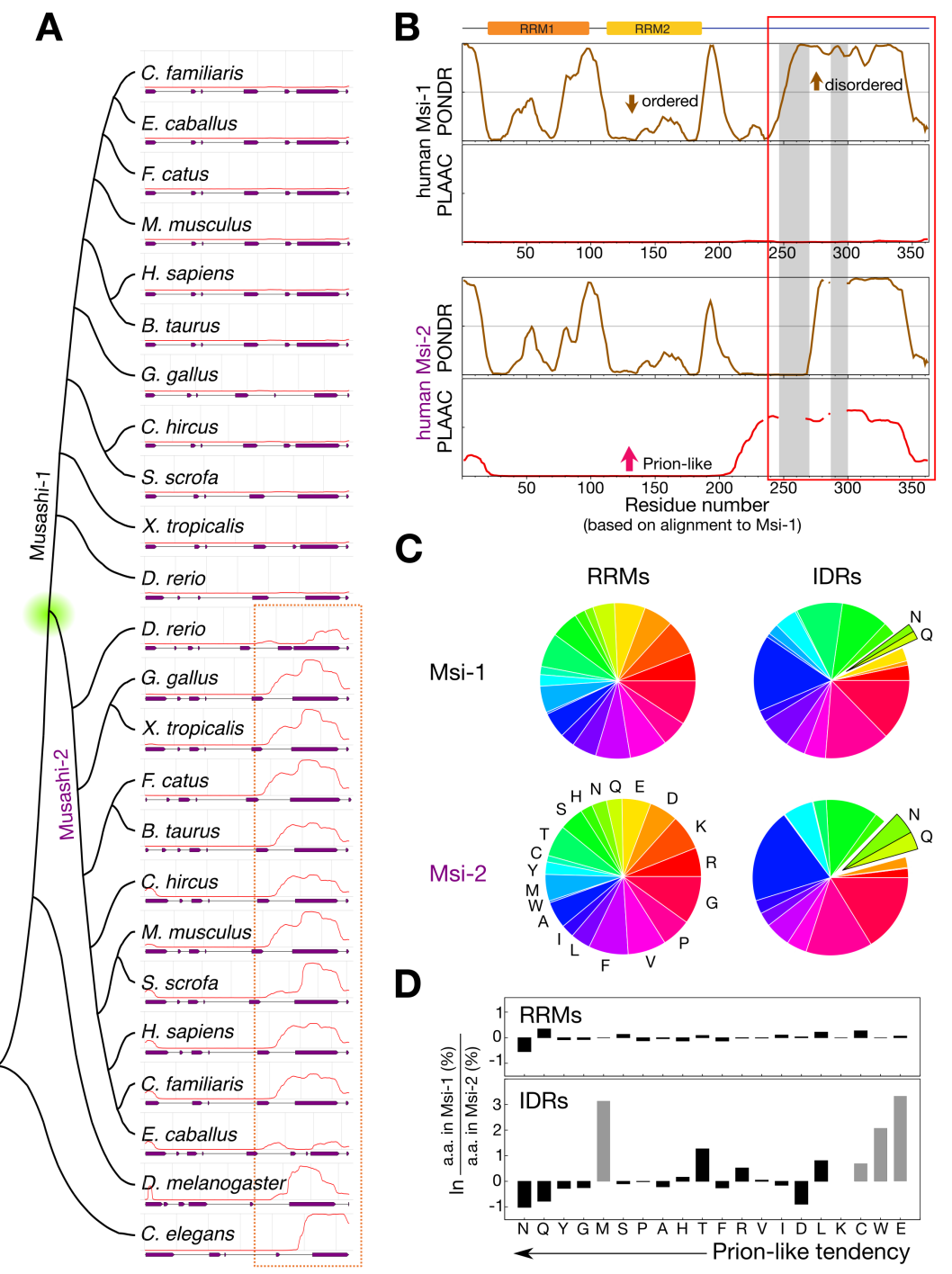

C
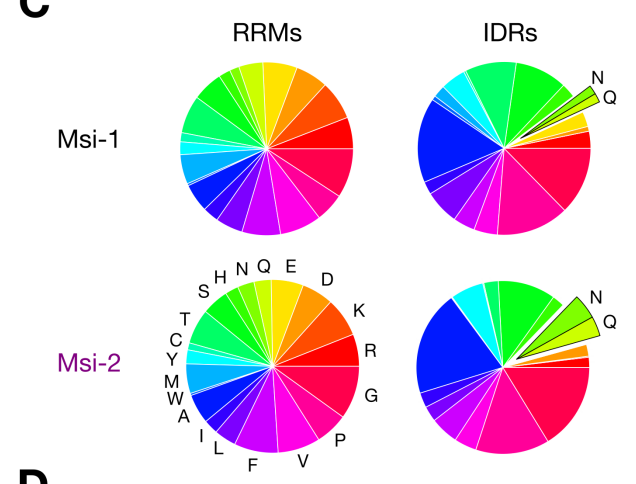

D



Fig. 1. Prion-like nature of Musashi protein homologs. (A) Phylogenetic tree (constructed using MEGA X (Kumar, et al. 2018)) of Musashi proteins in model organisms. The red lines indicate the PLAAC prion propensity scores (Lancaster, et al. 2014). The purple bars indicate regions predicted to be disordered by the VSL2 algorithm in PONDR (Obradovic, et al. 2005). The dashed box highlights the most prion-like regions. $(B)$ Disorder and prion-likeness analysis of human Musashi proteins. Residues are numbered according to the alignment with Musashi-1 and the gray shading indicates the regions that are missing in Musashi-2. The red-box contains the consensus intrinsically disordered regions (residues 237-362 of Musashi-1 and 235-328 of Musashi-2) considered in this study. (C), Amino acid pie charts of Musashi proteins in the model organisms listed in panel $(A)$. The RRMs and IDRs were defined as shown in panel $(B) .(D)$, Single amino-acid population differences in Musashi-1 and -2 with the amino acids sorted by prion-likeness (Lancaster, et al. 2014). Gray bars indicate amino-acids that appear fewer than ten times in the sequence. 

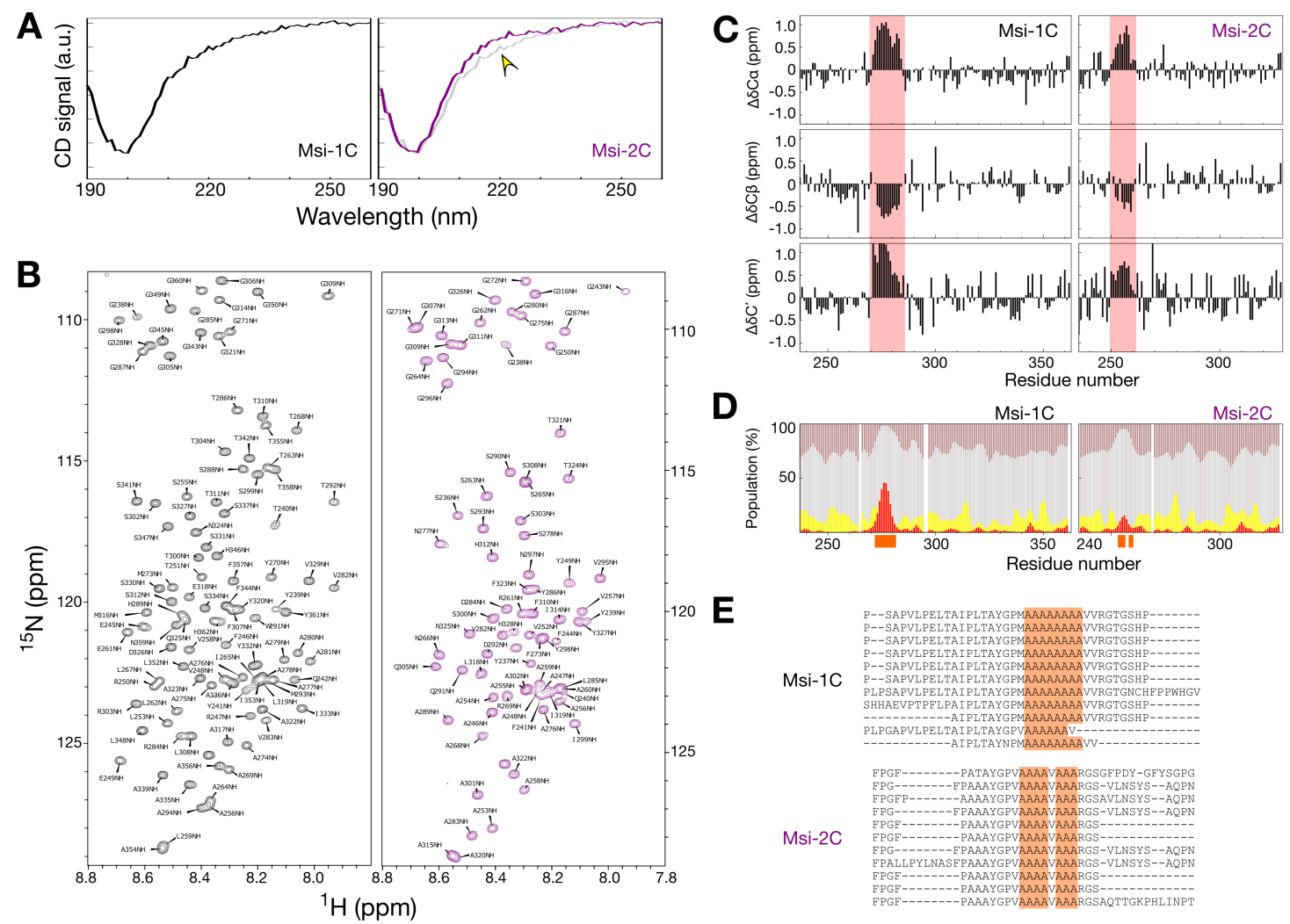

Fig. 2. The C-terminal domains of Musashi proteins are intrinsically disordered and have helix-forming polyalanine tracts. $(A)$ Circular dichroism (CD) spectra of the Cterminal domains of Musashi-1 (black; Msi-1C) and Musashi-2 (purple; Msi-2C). The spectrum of Msi-1C is also shown in the right panel (in gray) for comparison. The most obvious difference (at $\sim 220 \mathrm{~nm}$ ) is indicated with an arrow. $(B){ }^{15} \mathrm{~N}$-edited NMR HSQC spectra of Msi-1C and Msi-2C with resonance assignments. $(C)$ Secondary chemical shifts of $\mathrm{C} \alpha, \mathrm{C} \beta$, and carbonyl-carbon ( $\left.\mathrm{C}^{\prime}\right)$. The most pronounced deviations are highlighted in red. $(D)$ Stacked plots of the secondary structural populations derived from the chemical shifts using the $\delta 2 \mathrm{D}$ algorithm (Camilloni, et al. 2012). Red: $\alpha$-helix; yellow: $\beta$-sheet; gray: random coil; brown: polyproline II helix. The alanines in the polyalanine tracts are indicated by orange blocks on the $x$-axis. $(E)$ Multiple sequence alignment of Musashi homologs. The sequences are in the same order as shown in fig. $1 A$. The polyalanine stretch is highlighted in orange. 
A

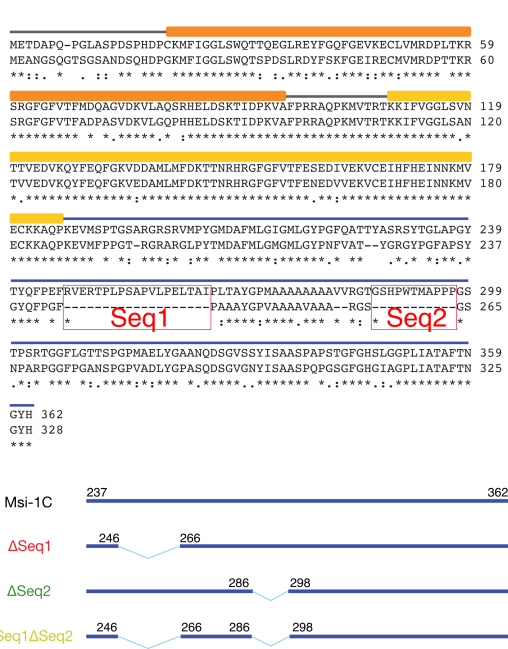

C

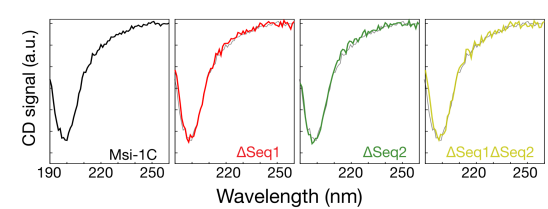

D
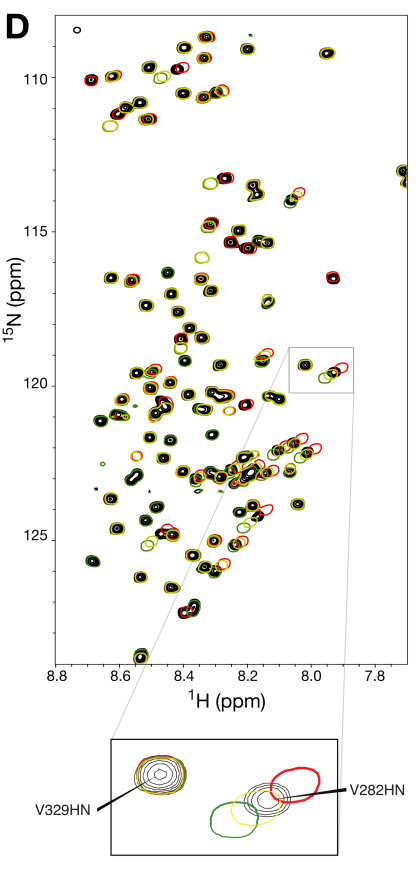

E

$\Delta$ Seq1-Msi-1C

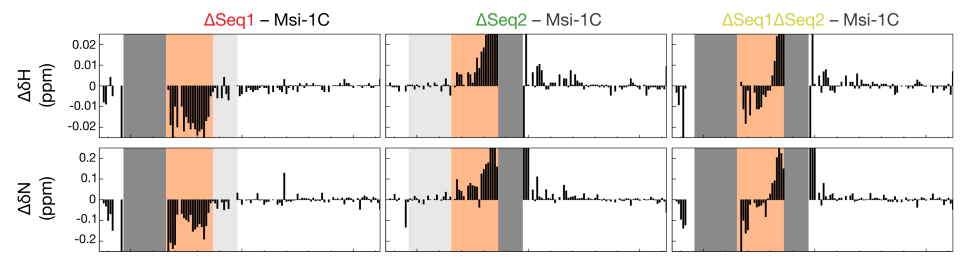

$\mathbf{F}$



G

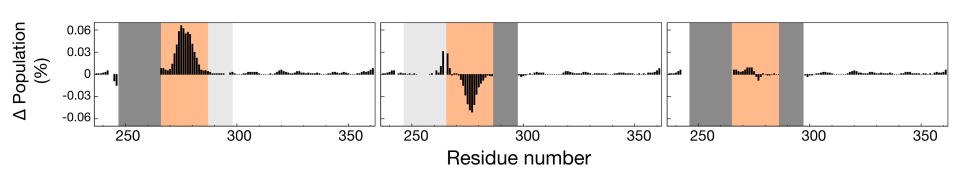

Fig. 3. The peptides flanking the polyalanine tract tune its $\alpha$-helical propensity. $(A)$ Sequence alignment of the human Musashi proteins. The main difference is the absence of the Seq1 and Seq2 regions in Musashi-2. (B) The designed constructs, with only the preserved residues indicated. $(C)$ Circular dichroism spectra of the different constructs (in black, red, green and yellow) overlaid on the wild type's (in gray). $(D)$ Overlaid NMR HSQC spectra (color-coded as in panel $(B)$ ), with an expanded view of overlapping (Val-329) and shifted (Val-282) cross-peaks. ( $E-G)$ Difference between the deletion constructs and the wild-type for $(E)$ proton $(\Delta \delta \mathrm{H})$ and nitrogen $(\Delta \delta \mathrm{N})$ chemical shifts, $(F)$ secondary chemical shift differences between $\mathrm{C} \alpha$ and $\mathrm{C} \beta ;(G) \delta 2 \mathrm{D}$ (Camilloni, et al. 2012) $\alpha$-helical propensity scores. The Seq1 and Seq2 regions are indicated in light gray if present, dark gray if missing; the polyalanine region is highlighted in orange. 
A

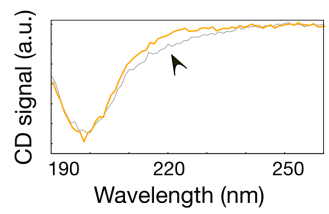

B



C

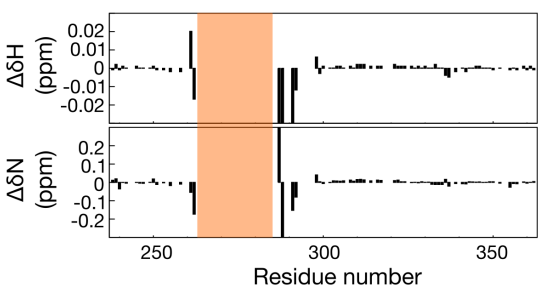

D

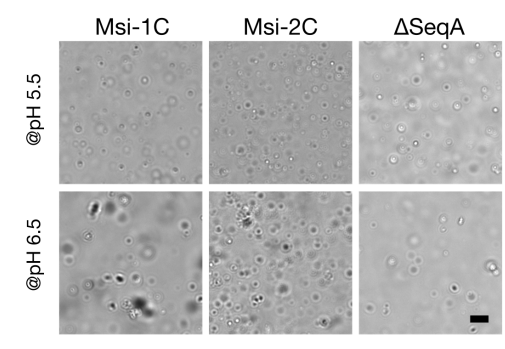

E
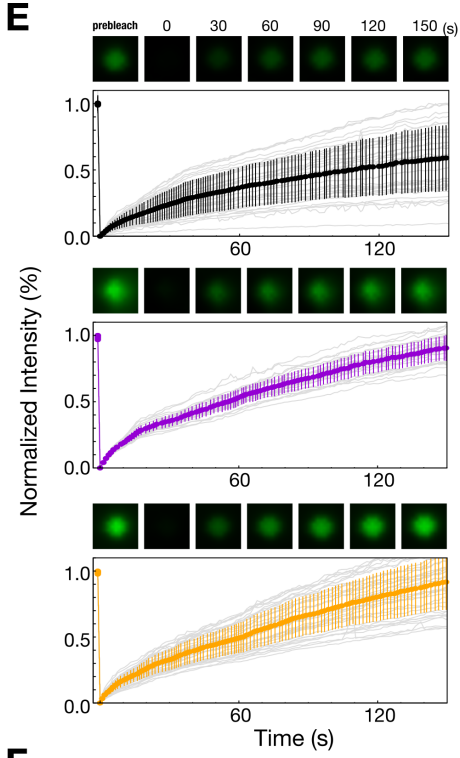

$\mathbf{F}$

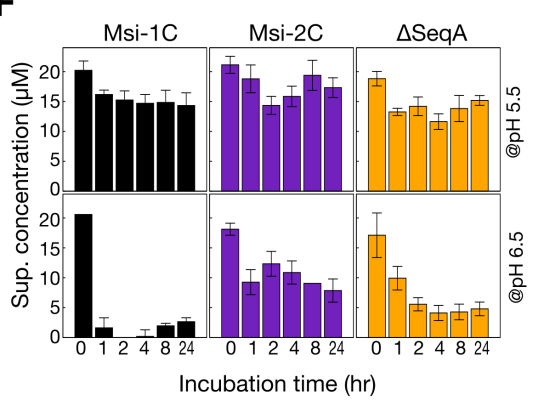

G

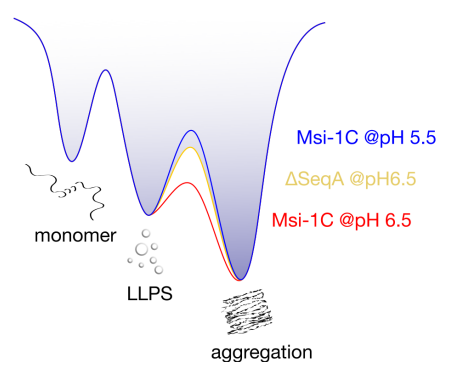

Fig. 4. The $\alpha$-helical region promotes self-assembly in Musashi-1's C-terminal domain (Msi-1C). Overlaid ( $A$ ) Circular dichroism, and $(B)$ NMR HSQC spectra of the $\triangle$ SeqA construct (orange) and Msi-1C (gray). (C) Chemical shift differences in the proton $(\Delta \delta \mathrm{H})$ and nitrogen $(\Delta \delta \mathrm{N})$ dimensions between $\Delta$ SeqA and Msi-1C. The polyalanine region is highlighted in orange. $(D)$ Light micrographs of Msi-1C, Msi-2C, and $\Delta$ SeqA at $\mathrm{pH} 5.5$ or 6.5 . Scale bar: $10 \mu \mathrm{m}$. (E) Fluorescence recovery after photobleaching (FRAP) results for Msi-1C and Msi-2C at pH 5.5. The colored lines represent the mean \pm standard deviation and the gray lines are individual recovery profiles. $(F)$ Precipitation assays of Msi-1C, Msi-2C, and $\Delta$ SeqA at $\mathrm{pH} 5.5$ and 6.5 after incubation at room temperature for different time periods. Supernatant concentrations were determined after centrifugation. $(G)$ Energy landscape representation of the results. 
A

Ala: 1.2

Leu: 1.0

Met: 1.0

Gln: 0.8

Rest: 0

B
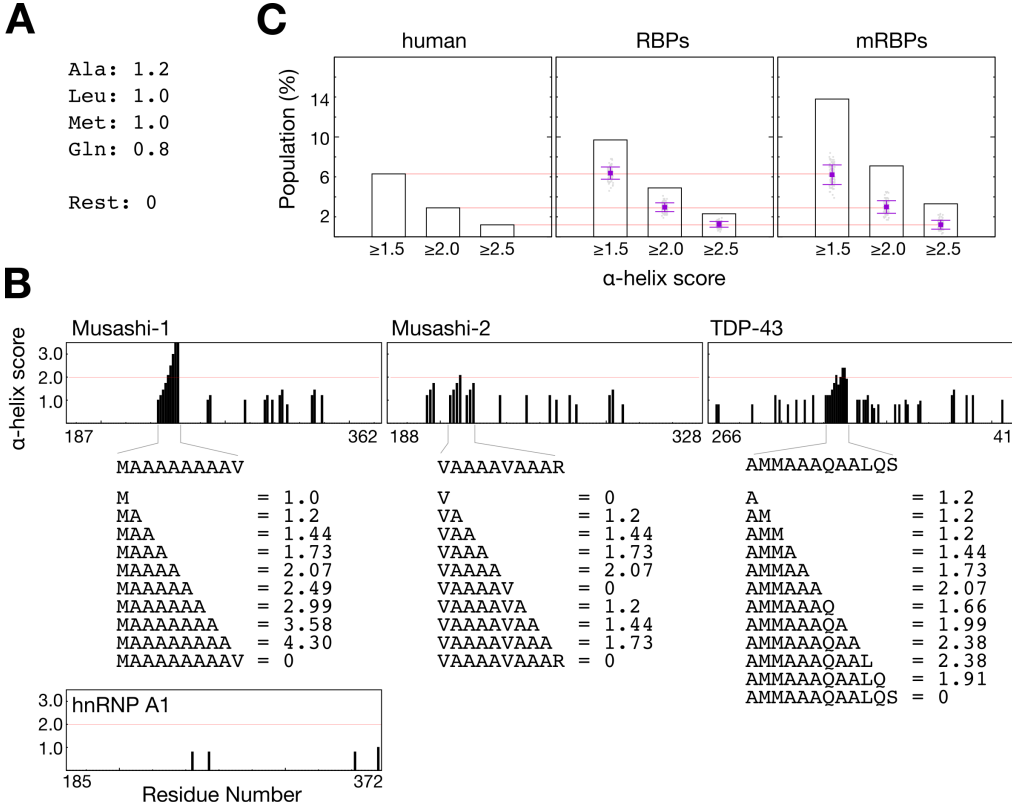

TDP-43

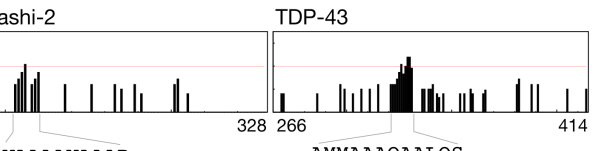

AMMAAAQAALQS

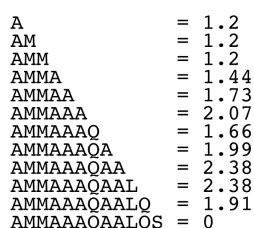

Fig. 5. Estimated prevalence of $\alpha$-helix-forming residue stretches in RNA-binding proteins (RBPs) and in the human proteome. $(A)$ Tentative scores are assigned to the amino acids according to their $\alpha$-helical propensity. $(B)$ Examples of how $\alpha$-helical propensity scores were calculated for continuous residue stretches of increasing length in proteins with $\alpha$-helix-forming regions (Musashi-1, Musashi-2, and TDP-43) or without (hnRNP A1). (C) Prevalence of IDRs with $\alpha$-helix scores greater than 2.5, 2.0, and 1.5 , in the human proteome, RBPs, and mRBPs. The point clouds, square points and error bars in the RBP and mRBP panels are the distribution, mean and standard deviation of the $\alpha$-helix scores of 100 random selections of sets of 1542 or 689 proteins (the RBP and mRBP sample sizes) from the human proteome (as negative controls). 

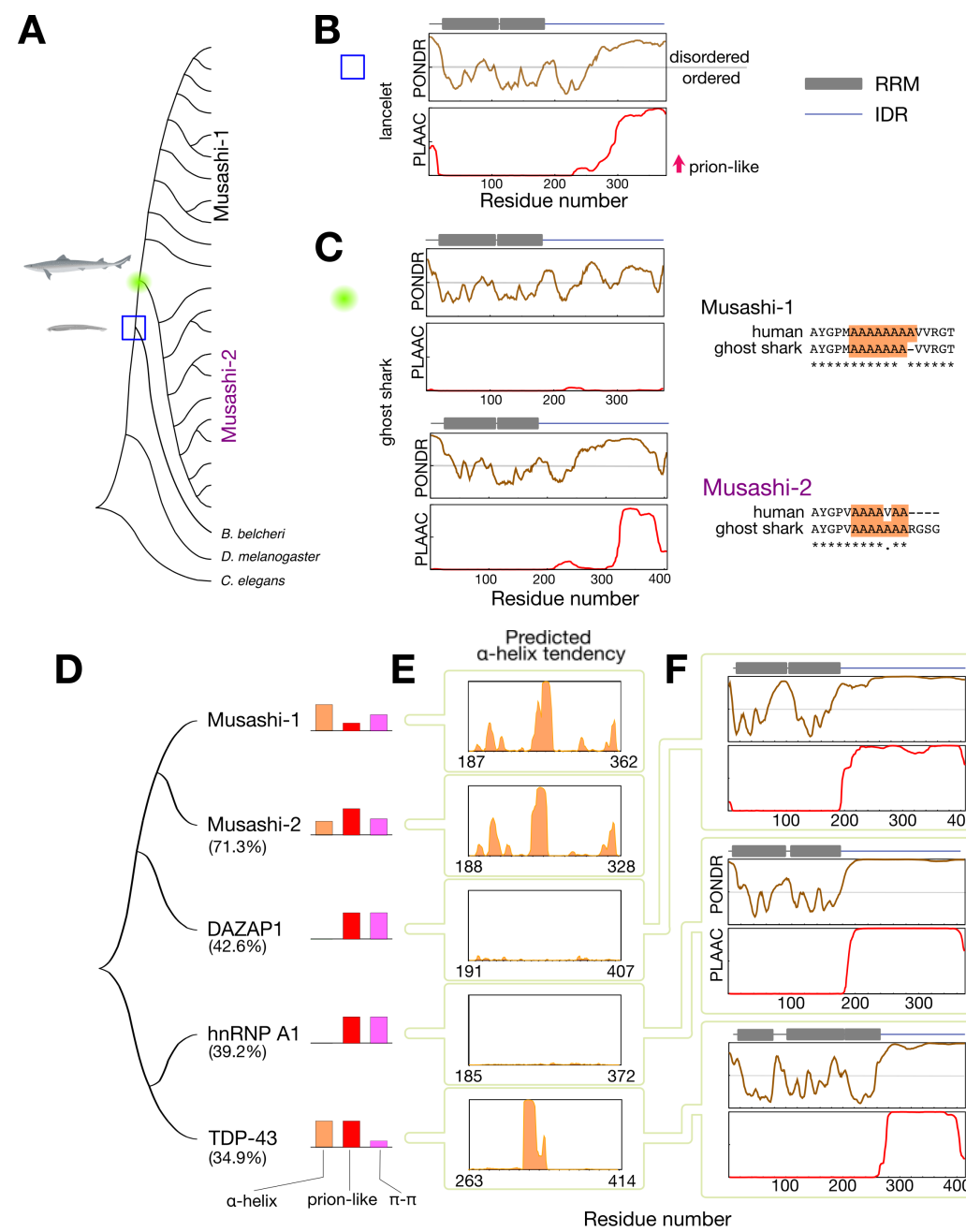

E $\quad \begin{gathered}\text { Predicted } \\ \text { a-helix tendency }\end{gathered} \quad \mathbf{F}$
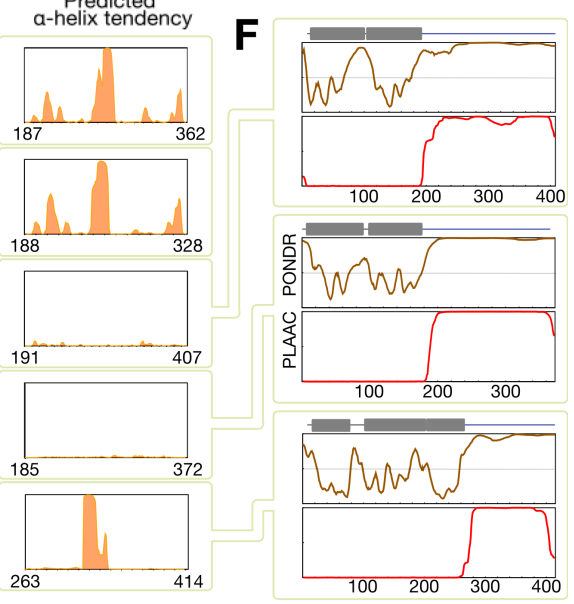

Fig. 6. Sequence analysis of the Musashi family in primitive model organisms and distantly related paralogs. $(A)$ Phylogenetic tree of the Musashi family as shown in fig. $1 A$ with the additional lineages analyzed: a primitive chordate (the lancelet, blue box, panel $(B))$ and a primitive vertebrate (the ghost shark, green circle, panel $(C)) .(B, C)$ PONDR sequence disorder and PLAAC prion-likeness scores of $(B)$ the lancelet (Buccinum belcheri) and $(C)$ the ghost shark (Callorhinchus milii). The RNA recognition motifs (based on PROSITE analysis (Sigrist, et al. 2010)) are indicated by gray squares. The alignment of the polyalanine regions (highlighted in orange) of human and ghost shark Musashi proteins are also shown. (D) Phylogenetic tree of Musashi paralogs with high sequence similarity found in the human proteome. The percentages shown are the levels of sequence similarity. The importance of different drivers of assembly in their IDRs is indicated by colored bars representing their $\alpha$ helical propensity (orange), prion-likeness (red), and number of aromatic residues (purple). (E) $\alpha$-helical propensity predicted using RaptorX (Yang, et al. 2018). $(F)$ Sequence disorder and prion-likeness for DAZAP-1, hnRNP-A1, and TDP-43 (c.f. fig $1 B$ for Musashi-1 and -2). 


\section{References:}

Alberti S, Hyman AA. 2021. Biomolecular condensates at the nexus of cellular stress, protein aggregation disease and ageing. Nat Rev Mol Cell Biol 22:196-213.

Alberti S, Saha S, Woodruff JB, Franzmann TM, Wang J, Hyman AA. 2018. A User's

Guide for Phase Separation Assays with Purified Proteins. J Mol Biol 430:4806-4820.

Bodenhausen G, Ruben DJ. 1980. Natural abundance N-15 NMR by enhanced heteronuclear spectroscopy. . Chem. Phys. Letters 69:185-189.

Camilloni C, De Simone A, Vranken WF, Vendruscolo M. 2012. Determination of secondary structure populations in disordered states of proteins using nuclear magnetic resonance chemical shifts. Biochemistry 51:2224-2231.

Chang SH, Chang WL, Lu CC, Tarn WY. 2014. Alanine repeats influence protein localization in splicing speckles and paraspeckles. Nucleic Acids Res 42:13788-13798. Chen HY, Lin LT, Wang ML, Tsai KL, Huang PI, Yang YP, Lee YY, Chen YW, Lo WL, Lan YT, et al. 2018. Musashi-1 promotes chemoresistant granule formation by PKR/elF2alpha signalling cascade in refractory glioblastoma. Biochim Biophys Acta Mol Basis Dis 1864:1850-1861.

Chen TC, Huang JR. 2020. Musashi-1: An Example of How Polyalanine Tracts Contribute to Self-Association in the Intrinsically Disordered Regions of RNA-Binding Proteins. Int J Mol Sci 21.

Chiou GY, Yang TW, Huang CC, Tang CY, Yen JY, Tsai MC, Chen HY, Fadhilah N, Lin CC, Jong YJ. 2017. Musashi-1 promotes a cancer stem cell lineage and chemoresistance in colorectal cancer cells. Sci Rep 7:2172.

Conicella AE, Zerze GH, Mittal J, Fawzi NL. 2016. ALS Mutations Disrupt Phase Separation Mediated by alpha-Helical Structure in the TDP-43 Low-Complexity CTerminal Domain. Structure 24:1537-1549.

Delaglio F, Grzesiek S, Vuister GW, Zhu G, Pfeifer J, Bax A. 1995. NMRPipe: a multidimensional spectral processing system based on UNIX pipes. J Biomol NMR 6:277-293.

Dominguez D, Freese P, Alexis MS, Su A, Hochman M, Palden T, Bazile C, Lambert NJ, Van Nostrand EL, Pratt GA, et al. 2018. Sequence, Structure, and Context Preferences of Human RNA Binding Proteins. Mol Cell 70:854-867 e859.

Fox RG, Park FD, Koechlein CS, Kritzik M, Reya T. 2015. Musashi signaling in stem cells and cancer. Annu Rev Cell Dev Biol 31:249-267.

Gebauer F, Schwarzl T, Valcarcel J, Hentze MW. 2020. RNA-binding proteins in human genetic disease. Nat Rev Genet.

Gerstberger S, Hafner M, TuschI T. 2014. A census of human RNA-binding proteins. Nat Rev Genet 15:829-845.

Greenland KN, Carvajal M, Preston JM, Ekblad S, Dean WL, Chiang JY, Koder RL, 
Wittebort RJ. 2018. Order, Disorder, and Temperature-Driven Compaction in a Designed Elastin Protein. J Phys Chem B 122:2725-2736.

Hardenberg M, Horvath A, Ambrus V, Fuxreiter M, Vendruscolo M. 2020. Widespread occurrence of the droplet state of proteins in the human proteome. Proc Natl Acad Sci U S A 117:33254-33262.

Hedges SB. 2002. The origin and evolution of model organisms. Nat Rev Genet 3:838849.

Hirota Y, Okabe M, Imai T, Kurusu M, Yamamoto A, Miyao S, Nakamura M, Sawamoto K, Okano H. 1999. Musashi and seven in absentia downregulate Tramtrack through distinct mechanisms in Drosophila eye development. Mech Dev 87:93-101. Hyberts SG, Frueh DP, Arthanari H, Wagner G. 2009. FM reconstruction of nonuniformly sampled protein NMR data at higher dimensions and optimization by distillation. J Biomol NMR 45:283-294.

Hyberts SG, Milbradt AG, Wagner AB, Arthanari H, Wagner G. 2012. Application of iterative soft thresholding for fast reconstruction of NMR data non-uniformly sampled with multidimensional Poisson Gap scheduling. J Biomol NMR 52:315-327. Itakura AK, Futia RA, Jarosz DF. 2018. It Pays To Be in Phase. Biochemistry 57:25202529.

Jacob F. 1977. Evolution and tinkering. Science 196:1161-1166.

Kato M, Han TW, Xie S, Shi K, Du X, Wu LC, Mirzaei H, Goldsmith EJ, Longgood J, Pei J, et al. 2012. Cell-free formation of RNA granules: low complexity sequence domains form dynamic fibers within hydrogels. Cell 149:753-767.

Kawashima T, Murakami AR, Ogasawara M, Tanaka K, Isoda R, Sasakura Y, Nishikata T, Okano H, Makabe KW. 2000. Expression patterns of musashi homologs of the ascidians, Halocynthia roretzi and Ciona intestinalis. Dev Genes Evol 210:162-165. Kharas MG, Lengner CJ, Al-Shahrour F, Bullinger L, Ball B, Zaidi S, Morgan K, Tam W, Paktinat $\mathrm{M}$, Okabe $\mathrm{R}$, et al. 2010. Musashi-2 regulates normal hematopoiesis and promotes aggressive myeloid leukemia. Nat Med 16:903-908.

Kjaergaard M, Brander S, Poulsen FM. 2011. Random coil chemical shift for intrinsically disordered proteins: effects of temperature and $\mathrm{pH}$. J Biomol NMR 49:139-149.

Kumar S, Stecher G, Li M, Knyaz C, Tamura K. 2018. MEGA X: Molecular Evolutionary Genetics Analysis across Computing Platforms. Mol Biol Evol 35:1547-1549. Kwon I, Kato M, Xiang S, Wu L, Theodoropoulos P, Mirzaei H, Han T, Xie S, Corden JL, McKnight SL. 2013. Phosphorylation-regulated binding of RNA polymerase II to fibrous polymers of low-complexity domains. Cell 155:1049-1060.

Lagadec C, Vlashi E, Frohnen P, Alhiyari Y, Chan M, Pajonk F. 2014. The RNA-binding protein Musashi-1 regulates proteasome subunit expression in breast cancer- and 
glioma-initiating cells. Stem Cells 32:135-144.

Lancaster AK, Nutter-Upham A, Lindquist S, King OD. 2014. PLAAC: a web and command-line application to identify proteins with prion-like amino acid composition. Bioinformatics 30:2501-2502.

Lee W, Tonelli M, Markley JL. 2015. NMRFAM-SPARKY: enhanced software for biomolecular NMR spectroscopy. Bioinformatics 31:1325-1327.

Lee W, Westler WM, Bahrami A, Eghbalnia HR, Markley JL. 2009. PINE-SPARKY: graphical interface for evaluating automated probabilistic peak assignments in protein NMR spectroscopy. Bioinformatics 25:2085-2087.

Levitt M. 1978. Conformational preferences of amino acids in globular proteins. Biochemistry 17:4277-4285.

Li HR, Chen TC, Hsiao CL, Shi L, Chou CY, Huang JR. 2018. The physical forces mediating self-association and phase-separation in the C-terminal domain of TDP-43. Biochim Biophys Acta 1866:214-223.

Li HR, Chiang WC, Chou PC, Wang WJ, Huang JR. 2018. TAR DNA-binding protein 43 (TDP-43) liquid-liquid phase separation is mediated by just a few aromatic residues. J Biol Chem 293:6090-6098.

Li L, Stoeckert CJ, Jr., Roos DS. 2003. OrthoMCL: identification of ortholog groups for eukaryotic genomes. Genome Res 13:2178-2189.

Li N, Yousefi M, Nakauka-Ddamba A, Li F, Vandivier L, Parada K, Woo DH, Wang S, Naqvi AS, Rao S, et al. 2015. The Msi Family of RNA-Binding Proteins Function Redundantly as Intestinal Oncoproteins. Cell Rep 13:2440-2455.

Lodish H, Berk A, Kaiser CA, Krieger M, Bretscher A, Ploegh H, Amon A, Martin KC. 2016. Molecular Cell Biology. New York: W. H. Freeman and Company.

Martin EW, Holehouse AS, Peran I, Farag M, Incicco JJ, Bremer A, Grace CR, Soranno A, Pappu RV, Mittag T. 2020. Valence and patterning of aromatic residues determine the phase behavior of prion-like domains. Science 367:694-699.

Molliex A, Temirov J, Lee J, Coughlin M, Kanagaraj AP, Kim HJ, Mittag T, Taylor JP. 2015. Phase separation by low complexity domains promotes stress granule assembly and drives pathological fibrillization. Cell 163:123-133.

Montalbano M, McAllen S, Puangmalai N, Sengupta U, Bhatt N, Johnson OD, Kharas MG, Kayed R. 2020. RNA-binding proteins Musashi and tau soluble aggregates initiate nuclear dysfunction. Nat Commun 11:4305.

Nakamura M, Okano H, Blendy JA, Montell C. 1994. Musashi, a neural RNA-binding protein required for Drosophila adult external sensory organ development. Neuron 13:67-81.

Obradovic Z, Peng K, Vucetic S, Radivojac P, Dunker AK. 2005. Exploiting heterogeneous sequence properties improves prediction of protein disorder. 
Proteins 61 Suppl 7:176-182.

Ohno S. 1970. Evolution by Gene Duplication: Springer.

Okano H, Imai T, Okabe M. 2002. Musashi: a translational regulator of cell fate. J Cell Sci 115:1355-1359.

Pace CN, Scholtz JM. 1998. A helix propensity scale based on experimental studies of peptides and proteins. Biophys J 75:422-427.

Patel A, Lee HO, Jawerth L, Maharana S, Jahnel M, Hein MY, Stoynov S, Mahamid J, Saha S, Franzmann TM, et al. 2015. A Liquid-to-Solid Phase Transition of the ALS Protein FUS Accelerated by Disease Mutation. Cell 162:1066-1077.

Piotto M, Saudek V, Sklenar V. 1992. Gradient-tailored excitation for single-quantum NMR spectroscopy of aqueous solutions. J Biomol NMR 2:661-665.

Polling S, Ormsby AR, Wood RJ, Lee K, Shoubridge C, Hughes JN, Thomas PQ, Griffin $M D$, Hill AF, Bowden Q, et al. 2015. Polyalanine expansions drive a shift into alphahelical clusters without amyloid-fibril formation. Nat Struct Mol Biol 22:1008-1015. Prestel A, Bugge K, Staby L, Hendus-Altenburger R, Kragelund BB. 2018.

Characterization of Dynamic IDP Complexes by NMR Spectroscopy. Methods Enzymol 611:193-226.

Sakakibara S, Imai T, Hamaguchi K, Okabe M, Aruga J, Nakajima K, Yasutomi D, Nagata T, Kurihara Y, Uesugi S, et al. 1996. Mouse-Musashi-1, a neural RNA-binding protein highly enriched in the mammalian CNS stem cell. Dev Biol 176:230-242.

Sakakibara S, Nakamura Y, Yoshida T, Shibata S, Koike M, Takano H, Ueda S, Uchiyama Y, Noda T, Okano H. 2002. RNA-binding protein Musashi family: roles for CNS stem cells and a subpopulation of ependymal cells revealed by targeted disruption and antisense ablation. Proc Natl Acad Sci U S A 99:15194-15199.

Sengupta U, Montalbano M, McAllen S, Minuesa G, Kharas M, Kayed R. 2018. Formation of Toxic Oligomeric Assemblies of RNA-binding Protein: Musashi in Alzheimer's disease. Acta Neuropathol Commun 6:113.

Shibata S, Umei M, Kawahara H, Yano M, Makino S, Okano H. 2012. Characterization of the RNA-binding protein Musashi1 in zebrafish. Brain Res 1462:162-173.

Sigrist CJ, Cerutti L, de Castro E, Langendijk-Genevaux PS, Bulliard V, Bairoch A, Hulo N. 2010. PROSITE, a protein domain database for functional characterization and annotation. Nucleic Acids Res 38:D161-166.

Smith RW, Anderson RC, Smith JW, Brook M, Richardson WA, Gray NK. 2011. DAZAP1, an RNA-binding protein required for development and spermatogenesis, can regulate mRNA translation. RNA 17:1282-1295.

Sundar J, Matalkah F, Jeong B, Stoilov P, Ramamurthy V. 2020. The Musashi proteins MSI1 and MSI2 are required for photoreceptor morphogenesis and vision in mice. J Biol Chem. 
Uversky VN. 2002. Natively unfolded proteins: a point where biology waits for physics. Protein Sci 11:739-756.

Uversky VN, Dunker AK. 2010. Understanding protein non-folding. Biochim Biophys Acta 1804:1231-1264.

Varadi M, Zsolyomi F, Guharoy M, Tompa P. 2015. Functional Advantages of Conserved Intrinsic Disorder in RNA-Binding Proteins. PLoS One 10:e0139731. Wiedner HJ, Giudice J. 2021. It's not just a phase: function and characteristics of RNA-binding proteins in phase separation. Nat Struct Mol Biol 28:465-473. Wilson EB. 1899. The Structure of Protoplasm. Science 10:33-45.

Xiang S, Kato M, Wu LC, Lin Y, Ding M, Zhang Y, Yu Y, McKnight SL. 2015. The LC Domain of hnRNPA2 Adopts Similar Conformations in Hydrogel Polymers, Liquid-like Droplets, and Nuclei. Cell 163:829-839.

Yang HT, Peggie M, Cohen P, Rousseau S. 2009. DAZAP1 interacts via its RNArecognition motifs with the $\mathrm{C}$-termini of other RNA-binding proteins. Biochem Biophys Res Commun 380:705-709.

Yang Y, Gao J, Wang J, Heffernan R, Hanson J, Paliwal K, Zhou Y. 2018. Sixty-five years of the long march in protein secondary structure prediction: the final stretch? Brief Bioinform 19:482-494.

Yoda A, Sawa H, Okano H. 2000. MSI-1, a neural RNA-binding protein, is involved in male mating behaviour in Caenorhabditis elegans. Genes Cells 5:885-895.

Zagrovic B, Bartonek L, Polyansky AA. 2018. RNA-protein interactions in an unstructured context. FEBS Lett 592:2901-2916. 